\title{
Using Optical Flow as Evidence for Probabilistic Tracking*
}

\author{
M.J. Lucena ${ }^{1}$, J.M. Fuertes ${ }^{1}$, N. Perez de la Blanca ${ }^{2}$, and A. Garrido ${ }^{2}$ \\ 1 Departamento de Informatica, Escuela Politecnica Superior, Universidad de Jaen, \\ Avda de Madrid 35, 23071 Jaen, Spain \\ \{mlucena, jmf $@$ @ujaen.es \\ 2 Departamento de Ciencias de la Computacion e Inteligencia Artificial, \\ ETSII. Universidad de Granada \\ C/ Periodista Daniel Saucedo Aranda s/n, \\ 18071 Granada, Spain \\ \{nicolas, agarrido\} @ugr.es
}

\begin{abstract}
In this paper, we presen t an obserntion model based on the Lucas and Kanade algorithm for computing optical flow, to trac k objects using particle filter algorithms. Although optical flow information enables us to know the displacement of objects present in a scene, it cannot be used directly to displace an object model since flow calculation techniques lack the necessary precision. In view of the fact that probabilistic tracking algorithms enable imprecise or incomplete information to be handled naturally, this model has been used as a natural means of incorporating flow information into the trac king.
\end{abstract}

\section{Introduction}

The probabilistic models applied to tracking [1][2][3][4] enable us to estimate the a posteriori probability distribution of the set of valid configurations for the object to be tracked, represented by a vector $\mathbf{X}$, from the set of measurements $\mathbf{Z}$ executed on the images of the sequence, $p(\mathbf{X} \mid \mathbf{Z})$. The likelihood in the previous instant is combined with a dynamical model giving rise to the a priori distribution in the current instant, $p(\mathbf{X})$. The relation betw een these distributions is given by Bay es' Theorem:

$$
p(\mathbf{X} \mid \mathbf{Z}) \propto p(\mathbf{X}) \cdot p(\mathbf{Z} \mid \mathbf{X})
$$

The distribution $p(\mathbf{Z} \mid \mathbf{X})$, known as the observation model, represents the probability of the measurements $\mathbf{Z}$ appearing in the images, assuming that a specific configuration of the model in the current instant is kno wn.

In this paper, an observation model is defined based on the optical flow of the sequence, chec king its validit y within a sheme of particle filter tracking.

\footnotetext{
* This work has been financed by grant TIC-2001-3316 from the Spanish Ministry of Science and Technology.
} 


\section{Dynamical model}

The model which represents the dynamical model of the object will provide an $a$ priori distribution on all the possible configurations in the instant $t_{k}, p\left(\mathbf{X}\left(t_{k}\right)\right)$, from the estimated distributions in the previous instants of time. In this paper, a second-order dynamical model has been used in which the two previous states of the object model are considered, and this is equivalent to taking a first-order dynamical model with a state vector for the instant $t_{k}$ of the form [5]

$$
\mathcal{X}_{t_{k}}=\left[\mathbf{X}_{t_{k-1}}, \mathbf{X}_{t_{k}}\right]^{T}
$$

The integration of the a priori distribution $p(\mathbf{X})$ with the set $\mathbf{Z}$ of the evidences present in each image, in order to obtain the a posteriori distribution $p(\mathbf{X} \mid \mathbf{Z})$, is obtained with Bayes' Theorem. This fusion of information can be performed, if the distributions are Gaussian, by using Kalman's Filter [1]. However, in general, the distributions involved in the process are normally not Gaussian and multimodal [3]. Sampling methods for modelling this type of distribution [6] have shown themselves to be extremely useful, and particle filter algorithms $[7,2,8,4]$ based on sets of weighted random samples, enable their propagation to be performed effectively.

\section{Observation model based on optical flow}

In order to build this model, we will use a technique derived from the LucasKanade algorithm[9] taking advantage of knowledge of the position of the flow discontinuities which predict the object model. Our hypothesis is based on the fact that the point $\mathbf{x}$ of the model outline is situated on the real outline of the object, and therefore we assume that the flow in a neighborhood of $\mathbf{x}$ shall only take two values: one on the inner part of the model, and the other on the outer part.

Let $\mathbf{x}=f\left(\mathbf{X}_{t_{k}} ; \mathbf{m}\right)$ (where $\mathbf{X}_{t_{k}}$ defines the specific configuration of the object model, and $\mathbf{m}$ is the parameter vector which associates each point within the model with a point on the image plane), a point belonging to the model outline in the instant $t_{k}$. Let $S$ be a neighborhood of $\mathbf{x}$ subdivided in $S_{i}$ and $S_{e}$ (corresponding respectively to the parts of the neighborhood which remain towards the interior and exterior of the outline of the model), and $\mathbf{d}\left(\mathcal{X}_{t_{k}}, \mathbf{m}\right)$ be calculated using the expression:

$$
\mathbf{d}\left(\mathcal{X}_{t_{k}}, \mathbf{m}\right)=f\left(\mathbf{X}_{t_{k}} ; \mathbf{m}\right)-f\left(\mathbf{X}_{t_{k-1}} ; \mathbf{m}\right)
$$

with $I^{(k-1)}$ and $I^{(k)}$ being the images corresponding to the instants of time $t_{k-1}$ and $t_{k}$. The system of equations [9] is therefore solved

$$
\left[\begin{array}{cc}
\sum_{S_{x}}\left(I_{x}^{(k-1)}\right)^{2} & \sum_{S_{x}} I_{x}^{(k-1)} I_{y}^{(k-1)} \\
\sum_{S_{x}} I_{x}^{(k-1)} I_{y}^{(k-1)} & \sum_{S_{x}}\left(I_{y}^{(k-1)}\right)^{2}
\end{array}\right]\left[\begin{array}{l}
f_{x} \\
f_{y}
\end{array}\right]=\left[\begin{array}{l}
-\sum_{S_{x}} I_{x}^{(k-1)} I_{t} \\
-\sum_{S_{x}} I_{y}^{(k-1)} I_{t}
\end{array}\right]
$$


In order to obtain the flow vector $\mathbf{f}_{S_{x}}=\left(f_{x}, f_{y}\right)$, where $S_{x}$ shall be $S_{i}$ or $S_{e}$, respectively, $I_{x}$ and $I_{y}$ are the spatial derivatives of the image and

$$
I_{t}(\mathbf{x})=I^{(k)}\left(\mathbf{x}+\mathbf{d}\left(\mathcal{X}_{t_{k}}, \mathbf{m}\right)\right)-I^{(k-1)}(\mathbf{x})
$$

In this way, two different flow estimations are obtained, $\mathbf{f}_{S_{i}}\left(\mathcal{X}_{t_{k}}, \mathbf{m}\right)$ and $\mathbf{f}_{S_{e}}\left(\mathcal{X}_{t_{k}}, \mathbf{m}\right)$, corresponding to the inner and outer area of the neighborhood of $\mathbf{x}$, respectively. The squared norm of the estimated flow vectors are then calculated, which is equivalent to obtaining the quadratic differences with the expected flow, which in this case equals zero:

$$
Z_{S_{i}}\left(\mathcal{X}_{t_{k}}, \mathbf{m}\right)=\left\|\mathbf{f}_{S_{i}}\left(\mathcal{X}_{t_{k}}, \mathbf{m}\right)\right\|^{2}, \quad Z_{S_{e}}\left(\mathcal{X}_{t_{k}}, \mathbf{m}\right)=\left\|\mathbf{f}_{S_{e}}\left(\mathcal{X}_{t_{k}}, \mathbf{m}\right)\right\|^{2}
$$

It should be noted that if the point $\mathbf{x}$ is really situated on a flow discontinuity, and the flow in $S_{i}$ coincides with $\mathbf{d}\left(\mathcal{X}_{t_{k}}, \mathbf{m}\right)$, the value of $Z_{S_{i}}$ must be close to zero and the value of $Z_{S_{e}}$ must be considerably greater. Using the following expression, these values may be combined and a value of $Z\left(\mathcal{X}_{t_{k}}, \mathbf{m}\right)$ may therefore be obtained:

$$
Z\left(\mathcal{X}_{t_{k}}, \mathbf{m}\right)=\left\{\begin{array}{cc}
\frac{Z_{S_{e}}\left(\mathcal{X}_{t_{k}}, \mathbf{m}\right)}{Z_{S_{e}}\left(\mathcal{X}_{t_{k}}, \mathbf{m}\right)+Z_{S_{i}}\left(\mathcal{X}_{t_{k}}, \mathbf{m}\right)} & \text { if } Z_{S_{e}}\left(\mathcal{X}_{t_{k}}, \mathbf{m}\right) \neq Z_{S_{i}}\left(\mathcal{X}_{t_{k}}, \mathbf{m}\right) \\
1 / 2 & \text { if } Z_{S_{e}}\left(\mathcal{X}_{t_{k}}, \mathbf{m}\right)=Z_{S_{i}}\left(\mathcal{X}_{t_{k}}, \mathbf{m}\right)
\end{array}\right.
$$

The value of $Z\left(\mathcal{X}_{t_{k}}, \mathbf{m}\right)$ satisfies the following properties:

$-0 \leq Z\left(\mathcal{X}_{t_{k}}, \mathbf{m}\right) \leq 1$

- If $Z_{S_{e}}\left(\mathcal{X}_{t_{k}}, \mathbf{m}\right) \gg Z_{S_{i}}\left(\mathcal{X}_{t_{k}}, \mathbf{m}\right)$, then $Z\left(\mathcal{X}_{t_{k}}, \mathbf{m}\right) \rightarrow 1$, which indicates that the adjustment is much better in $S_{i}$ than it is in $S_{e}$, and therefore the point must be situated exactly in a flow discontinuity, in which the inner area coincides with the displacement predicted by the model.

- If $Z_{S_{e}}\left(\mathcal{X}_{t_{k}}, \mathbf{m}\right) \ll Z_{S_{i}}\left(\mathcal{X}_{t_{k}}, \mathbf{m}\right)$, then $Z\left(\mathcal{X}_{t_{k}}, \mathbf{m}\right) \rightarrow 0$. The adjustment is worse in the inner area than it is in the outer area, and therefore the estimated flow does not match the model's prediction.

- If $Z_{S_{e}}\left(\mathcal{X}_{t_{k}}, \mathbf{m}\right)=Z_{S_{i}}\left(\mathcal{X}_{t_{k}}, \mathbf{m}\right)$, then the adjustment is the same in the inner area as it is in the outer area, and therefore the flow adequately matches the displacement predicted by the model, but it is impossible to guarantee that it is situated on a flow discontinuity. In this case, $Z\left(\mathcal{X}_{t_{k}}, \mathbf{m}\right)=1 / 2$.

It is possible that some of the areas $S_{i}$ or $S_{e}$ lack enough structure to give a good flow estimate. In this paper, we have used the inverse condition number [10] of the coefficient matrix in the expression (2), $R=\lambda_{\min } / \lambda_{\max }$, in order to check the stability of the equation system, so that if it is too small $\left(<10^{-10}\right)$, it is necessary to discard the flow values obtained, and therefore $Z\left(\mathcal{X}_{t_{k}}, \mathbf{m}\right)=1 / 2$.

We shall consider that the presence probability of the measurements obtained for the image, since they have been caused by the point of the outline 


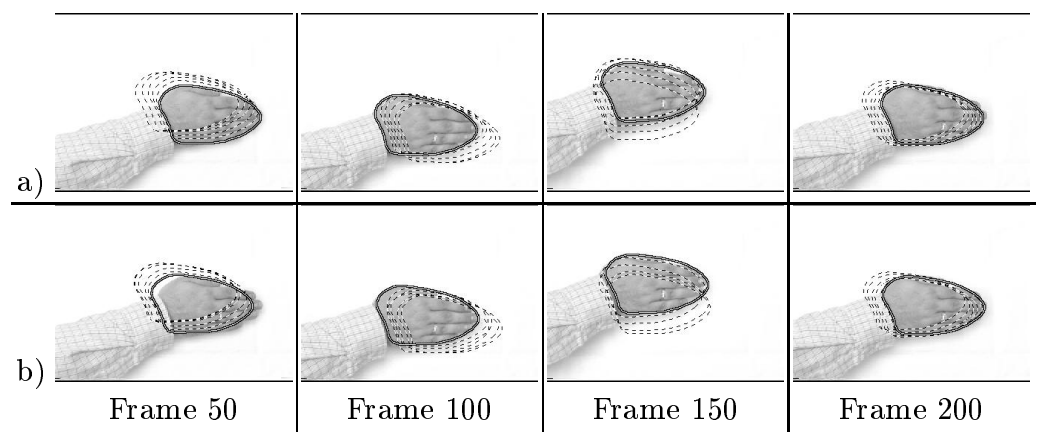

Fig. 1. a) Results obtained with the observation model for the contour normals. b) Results obtained with the observation model based on optical flow restrictions. The distribution average appears in solid line in the current frame, and the averages in some previous frames appear in dashed line.

corresponding to the vector $\mathbf{m}$ of the sample in question, defined by the vector $\mathcal{X}_{t_{k}}$, must be proportional to the function $Z\left(\mathcal{X}_{t_{k}}, \mathbf{m}\right)$ obtained previously,

$$
p\left(\mathbf{Z} \mid \mathcal{X}_{t_{k}}, \mathbf{m}_{i}\right) \propto Z\left(\mathcal{X}_{t_{k}}, \mathbf{m}_{i}\right)
$$

and that, given the independence between the different points of the outline,

$$
p\left(\mathbf{Z} \mid \mathcal{X}_{t_{k}}\right) \propto \prod_{i} Z\left(\mathcal{X}_{t_{k}}, \mathbf{m}_{i}\right)
$$

\section{Experiments}

In order to check the validity of the observation model proposed, it has been they were incorporated into the CONDENSATION algorithm [2], and its performance was compared with that of the observation model based on normals as proposed in $[5]$.

For the experiments, two image sequences were used, lasting 10 seconds, with 25 frames per second, $320 \times 240$ pixels, 8 bits per band and pixel, corresponding to the movement of a hand over an uniform and non uniform background. Results can be downloaded from http://wwwdi.ujaen.es/ mlucena/invest.html

\subsection{Tracking an object over an uniform background}

In order to model the hand, an outline model based on a closed spline with 10 control points and a Euclidean similarity deformation space were used. A second-order dynamical model was used in which the object tended to maintain velocity, and a preliminary tracking was carried out of the hand by using the gradient observation model along the contour normals. With the data obtained, 
the multidimensional learning method proposed in $[11,5]$ was used to determine the dynamic parameters.

For the observation model based on contour normals, 20 normals were sketched for each sample. The observation model was applied with parameters $\alpha=$ 0.025 and $\sigma=3$, incorporated into the Condensation algorithm with 200 samples. The initialization was carried out manually, indicating the position of the object in the first frame. Figure 1.a shows the weighted average of the distribution obtained.

The observation model based on optical flow was used on the same 20 points along the outline, defining a neighborhood for each point of $7 \times 7$ pixels. In order to calculate the spatial derivatives, each frame was convolved with two Gaussian derivative masks, with vertical and horizontal orientations respectively, with $\sigma=1.0$. The number of samples was also 200, and the results obtained are shown in Figure 1.b.

\subsection{Tracking an object over a non uniform background}

In this case, the parameters of the dynamic model were adjusted manually for the first 50 frames, and these were used to learn dynamics and to execute an initial tracking of the sequence, using CONDENSATION with the observation model for the contour normals. From the results obtained, and using the same learning method as in the previous experiment, the dynamic parameters were determined.

In order to use the observation model based on contour normals, 18 normals were sketched to each outline. The number of samples was still 200, and the parameters for the observation model in this case were $\sigma=3$ and $\alpha=0.055$. The results are shown in Figure 2.a.

The same parameters were used in the observation model based on optical flow as in the previous sequence, that is to say, spatial derivatives from Gaussian derivative masks with $\sigma=1.0$, and neighborhoods of $7 \times 7$ pixels for each point. The results obtained with 200 samples are shown in Figure 2.b.

\section{Discussion and conclusions}

The observation model based on contour normals behaves appropriately in the two sequences (Figures 1.a and 2.a). At no time does the tracker lose the object, although it does have problems with noise in the sequence due to the presence of clutter.

For the first sequence, the model based on optical flow, although never completely losing the object, does have problems focusing exactly on its outline. This is due to the absence of texture on the outer part of the object. However, for the second sequence, the presence of a background with a lot of noise is not only irrelevant but also favors the good behavior operation of the observation model.

The results obtained suggest that the observation models based on optical flow are, in a way, complementary to those based on gradient along normals. The presence of clutter constitutes a source of noise for the first models, while favoring the good behavior operation of models based on flow. 


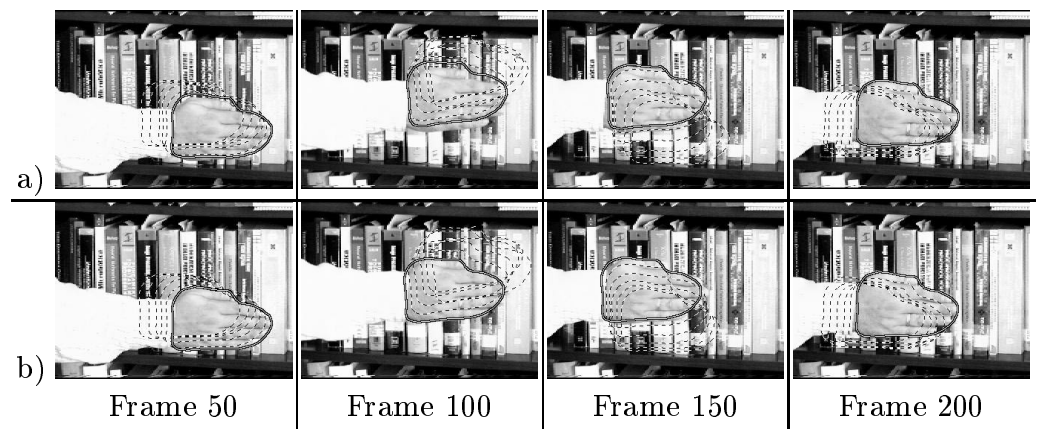

Fig. 2. a) Results obtained with the observation model for the contour normals. b) Results obtained with the observation model based on optical flow restrictions. The distribution average appears in solid line in the current frame, and the averages in some previous frames appear in dashed line.

\section{References}

1. R.E. Kalman, "A new approach to linear filtering and prediction problems," Transactions of the ASME-Journal of Basic Engineering, vol. 82, no. Series D, pp. 35-45, 1960 .

2. M. Isard and A. Blake, "Condensation - conditional density propagation for visual tracking," International Journal on Computer Vision, 1998.

3. J. Deutscher, A. Blake, B. North, and B. Bascle, "Tracking through singularities and discontinuities by random sampling," in Proceedings of International Conference on Computer Vision, 1999, vol. 2, pp. 1144-1149.

4. J. Sullivan, A. Blake, M. Isard, and J. MacCormick, "Bayesian object localisation in images," International Journal of Computer Vision, vol. 44, no. 2, pp. 111-135, 2001.

5. A. Blake and M. Isard, Active Contours, Springer, 1998.

6. A. Gelfand and A. Smith, "Sampling-based approaches to computing marginal densities," Journal of the American Statistical Association, vol. 85, no. 410, pp. 398-409, 1990.

7. M. Isard and A. Blake, "Contour tracking by stochastic propagation of conditional density," in Proceedings of European Conference on Computer Vision, Cambridge, UK, 1996, pp. 343-356.

8. J. Deutscher, A. Blake, and I. Reid, "Articulated body motion capture by annealed particle filtering," in Proceedings of IEEE Conference on Computer Vision and Pattern Recognition (CVPR), 2000.

9. B. Lucas and T. Kanade, "An iterative image registration technique with an application to stereo vision," in Proceedings of DARPA IU Workshop, 1981, pp. $121-130$.

10. W. H. Press, S. A. Teulosky, W. T. Vetterling, and B. P. Flannery, Numerical Recipes in C, Second Edition, Cambridge University Press, 1992.

11. A. Blake, M. Isard, and D. Reynard, "Learning to track the visual motion of contours," Journal of Artificial Intelligence, vol. 78, pp. 101-134, 1995. 